\title{
A Review on Different Micromixers and its Micromixing within Microchannel
}

\author{
Supriya Gambhire ${ }^{{ }^{*}}$, Nishigandha Patel ${ }^{\dagger}$, Gajanan Gambhire ${ }^{\ddagger}$ and Sachine Kale ${ }^{\dagger}$

\begin{abstract}
${ }^{\dagger}$ Mechanical Engineering Department, Savitribai Phule Pune University, MIT College of Engineering, Pune, India
*Industrial and Production Engineering Department, An Autonomous Institute affiliated to Savitribai Phule Pune University, Vishwakarma Institute of Technology, Pune, India
\end{abstract}

Accepted 02 March 2016, Available online 15 March 2016, Special Issue-4 (March 2016)

\begin{abstract}
Micromixing technology has experienced rapid development in the past few years. Micromixers represent one of the essential components in integrated microfluidic systems for chemical, biological and medical applications In general, macro-scale mixing is achieved with turbulence while mixing in micro-scale is done by diffusion. This is due to low Reynolds number i.e laminar behavior of the flow in micro channels. Micro-mixer with obstacles located on the channel wall as well as waveform configurations are used to enhance mixing in the channel, so as to reduce the mixing length. Micro channels with different geometric layout and with different shapes and sizes of obstacles such as rectangular, triangular and semicircular enhance their mixing length. The triangular obstacles within the $T$ as well as $Y$ channel gave minimum mixing length for the same distance between the obstacles. The commercial computational fluid dynamics tool for micro-fluidics, known as Comsol multyphysics, is used to study the effect of different configuration on pressure drop as well as to study the mixing of two fluids in different configurations. Computational fluid dynamics (CFD) is often used to rigorously examine the influence of the shape of microchannels on mass transport phenomena in the flow field. Fabrication of micro channels is done on PDMS and Photochemical Machining. The fabrication of microfluidic by PDMS is easier and more flexible than in silicon or glass. The use of PDMS as a material reduces the time, complexity, and cost of prototyping. It is observed that mixing length required for Plain channel without obstacle is more than channel with obstacles. Because due to obstacles, turbulence is created at tip of obstacles which increases mixing rate.
\end{abstract}

Keywords: Micromixing, Microchannel, PDMS, Photochemical Machining.

\section{Introduction}

Micromixer was considered as an important component in microfluidic devices than the micropump, microactuator and microvalve, because the mixing time decides the analysis speed of the devices. A well-designed micromixer has rapid mixing and Compact in size .

The aim of microfluidic mixing is to achieve a thorough and rapid mixing of multiple samples in microscale devices. In such devices, sample mixing is essentially achieved by enhancing the diffusion effect between the different species flows. On the basis of principles exploited to induce mixing at the microscale, micromixers are generally classified as passive or active. Where for active micromixers, an external energy force is applied to perturb the sample species, or passive, where the contact area and contact time of the species samples are increased through speciallydesigned microchannel configurations .

The shape of microchannels is another important design variable to achieve the desired performance.

*Corresponding author: Supriya Gambhire
Since most microchannels are designed by trial and error, a systematic shape design method needs to be established. The main advantage of microfluidic devices is to investigate complex hydrodynamic effects using small quantities of liquids. However, the benefit of microfluidics has been proven in almost all scientific applications and consequently more research laboratories are focusing their activity on this area. In general, microfluidic devices are typically composed of a series of planar channels involving either sudden changes in direction ( $\mathrm{T}$ or Y sharp shapes, serpentines or abrupt variations of the flow area contraction or extension). The flows in these configurations are characterized by high deformation rates and small Reynolds numbers, even though the characteristic viscosities of the samples have the same order of magnitude as water.

\section{Classification of Micromixers}

Micromixers can be classified as passive and active. Active mixing requires more complex structures and therefore, a more complicated fabrication process. In 
order to enhance the passive mixing capability, it is essential to increase the contact interface between two solutions. Methods that have been effectively developed for this purpose are split and recombine lamination, recirculation (recycle flow) and chaotic advection.

Each of these micromixers has different capacity, mixing speed and operating requirements. For example, an active micromixer requires power input in order to effect mixing in the device while a passive micromixer achieves mixing with the applied pressure for fluid motion.

\subsection{Passive Micromixers}

A Passive micromixer depends on the mass transport phenomena provided by molecular diffusion and chaotic advection. These devices are designed with a channel geometry that increases the surface area between the different fluids and decreases the diffusion path. Mixed phase passive micromixers can be categorized as:

\subsubsection{T or Y Shaped Micromixers}

$\mathrm{T}$ or $\mathrm{Y}$ shaped channel micromixers are easiest and most basic designs. The mixing process in this type of micromixer is obtained by guiding the two liquids to be mixed in contact area when flow through channel. In this, mixing only depends on diffusion of the species at the interface between the two liquids, hence the mixing is rather slow and a long mixing channel is required. Further reduction of the mixing time could be achieved by using a high flow rate, hence high Re, where a chaotic flow is expected. Reduced mixing path in a Tshaped micromixer is obtained by a simple narrowing of the mixing channel and therefore shortening of the diffusion length.

\subsubsection{Parallel Lamination Micromixer}

The concept of $\mathrm{T}$ and $\mathrm{Y}$ shaped micromixers can be improved by using more complicated designs that split the inlet main streams into $\mathrm{n}$ sub-streams and then rejoin them to form a laminate stream (Fig. 2.1).

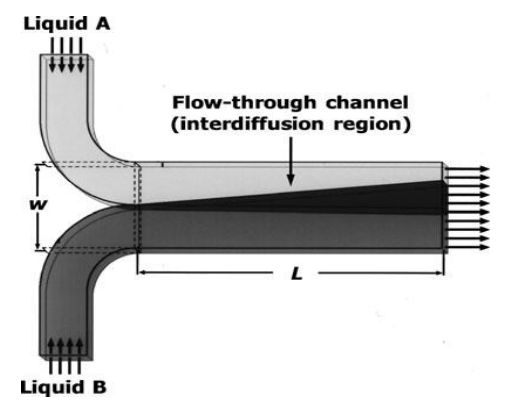

Fig. 2.1 T-shaped micromixer with two input

Where, $\mathrm{L}$ and $\mathrm{w}$ represent the length and width of the mixing channel, respectively. This type of micromixer enhances the mixing process by decreasing the diffusion length and increasing the contact surface area between the two fluids.

The swirling of the fluid flow obtained at higher $\mathrm{Re}$ number results in better dispersion of the fluid within the channel volume and hence an improvement in the mixing quality.

\subsubsection{Sequential Lamination Micromixer}

Sequential lamination micromixers is also called as split and recombine (SAR) micromixers, rely on an exponential increase in the contact surface area and decrease in the length path to achieve a shorter mixing time. The difference is that, lamination in sequential lamination micromixers is obtained by sequential processes of splitting and rejoining the fluids. However, in order to achieve exponential sequential lamination, three steps are typically required: flow splitting, flow recombination, and flow rearrangement.

\subsubsection{Flow Focusing Micromixer}

The basic design for hydrodynamic focusing is a long microchannel with three inlets. Typically, focusing enhanced micromixers focus the sample flow only in the horizontal dimension.

\subsubsection{Chaotic Advection Micromixers}

Advection is the transport of a substance within a moving fluid. In the micromixers discussed above, advection generally occurs in the direction of the flow; hence it has no effect on the transversal transport of the substance. However, advection in other directions, so-called chaotic advection, can generate a transverse component of flow. These generated transverse flow components cause an exponential growth of the interfacial area and a corresponding decrease in the striation thickness, which can significantly improve mixing.

Typically such type of micromixer can provide an effective mixing only for high $\mathrm{Re}$ in the range of few hundreds. These micromixers are generally described using dimensionless number, the Dean number (De):

$\mathrm{De}=\operatorname{Re} \sqrt{\frac{\mathrm{Dh}}{\mathrm{R}}}$

Where, $\mathrm{R}$ represent the channel curvature. In order to provide an efficient mixing De must be greater than 140 .

\subsection{Active Micromixers}

Active micromixers rely on an external energy input to introduce perturbation within the fluid streamlines to achieve mixing. Therefore, they are categorized with respect to the type of external perturbation energy:

\subsubsection{Pressure Field Disturbance}

In a pressure field disturbance, a microfluidic mixer based on the same concept of fluid discretization and operated by two vortex micropumps. The discretized 
fluid constituted of discrete volumes of liquids to be mixed, is then pumped into an expansion chamber to increase the interfacial surface area between the volumes. The flow in the micromixer had a Re of 30.

\subsubsection{Electrokinetic Disturbance}

Elecrokinetic disturbance micromixers use fluctuating electric field to induce mixing in microfluidic channels or chambers. It causes rapid stretching and folding of the fluids interfaces that are able to stir the fluid stream in highly laminar flow $(\operatorname{Re}<1)$. EKI mixing effectiveness can be enhanced by combining its action with a passive micromixing strategy using channel geometries that induce secondary flow.

\subsubsection{Dielectrophoretic Disturbance}

Dielectrophoresis is a phenomenon in which polarization of particle is induced by a nonuniform electric field. Polarized particles can move towards or away from the electrodes in response to the electrical field applied. A synergistic effect between the movement of the particles and the geometry of the channel causes the creation of chaotic advection that causes the mixing of the fluid surrounding the particle.

\subsubsection{Electrowetting Shaking}

The movement of liquid droplets can generate flow patterns within the fluid and enhance the mixing of species inside the droplets. An active way to induce mixing in droplets is represented by electrowetting on dielectrics (EWOD), or simply electrowetting. Droplets containing different species can be electrically actuated to coalesce using electrowetting effect. After the coalescence, diffusion begins in the droplet and mixing of the two fluid species occurs. However, this passive like mixing is rather slow. To speed up the mixing process, different authors have proposed the use of electrowetting to shake, split and merge the droplets in order to create recirculating patterns that increase the interfacial area between the two liquids to be mixed. The droplets act as virtual mixing chambers and mixing occurs by oscillating the droplet across a number of electrodes at various frequencies. It can achieve mixing in a much more confined space than channel based mixing .

\subsubsection{Magneto-Hydrodynamic Disturbance}

Magneto-hydrodynamic (MHD) disturbance relies on the induction of Lorentz body forces in an electrolyte solution. MHD devices utilize an array of electrodes deposited in the channel walls to create current flows within the fluid to be mixed, in the presence of an alternate potential difference on the electrode pair. By coupling the generated electric field with a magnetic field, Lorentz body force could be generated. The complex flow field generated deformations and stretched the material interface, enhancing the mixing.

\subsubsection{Ultrasound Disturbance}

Mixing can be achieved by means of acoustic stirring created by ultrasonic waves. Ultrasounds are introduced into the channel by integrated piezoelectric ceramic transducers. The ultrasonic action causes an acoustic stirring of the fluid perpendicular to the flow direction and leads to an enhancement of the mixing inside the microfluidic channel or chamber. A turbulent like mixing was achieved at Re. Air bubbles can be introduced in a microfluidic mixer in order to enhance the mixing process. The surface of an air bubble in a liquid medium exposed to a sound field can act as a vibrating membrane. The membrane vibration causes a bulk fluid movement at the air-liquid interface. This effect, known as cavitation microstreaming, has been applied in microfluidic micromixers using a single bubble or an array of bubbles $<1$.

\section{Principles and Terminology of Fluid Mixing}

There are several words which can be used with the same meaning such as mixing and stirring. That is, the term 'mixing' means a physical process where both the stirring and the diffusion occur simultaneously. Here, the word stirring means the advection of material blobs subjected to mixing without diffusive action. In other words, we can say that good mixing of low diffusivity materials occurs in two stages; stirring in the first stage and diffusion in the second stage.

Table 3.1 Summary of the main features of various means for micromixing and their overall rank for use in microfluidic devices as a mixer

\begin{tabular}{|c|c|c|c|c|c|}
\hline 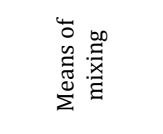 & 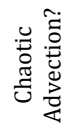 & 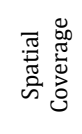 & 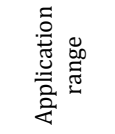 & 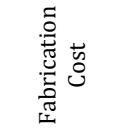 & 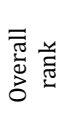 \\
\hline $\begin{array}{l}\text { Hydrodynami } \\
\text { c focusing }\end{array}$ & No & Local & Broad & High & 4 \\
\hline $\begin{array}{l}\text { Alternate } \\
\text { injection }\end{array}$ & No & Local & Broad & Moderate & 3 \\
\hline $\begin{array}{l}\text { Geometry } \\
\text { effect }\end{array}$ & Yes & Global & Broad & Moderate & 2 \\
\hline $\begin{array}{l}\text { Electrokinetic } \\
\text { method }\end{array}$ & Yes & Global & Moderate & High & 3 \\
\hline $\begin{array}{c}\text { Droplet } \\
\text { mixing }\end{array}$ & Yes & Global & Broad & Low & 1 \\
\hline $\begin{array}{l}\text { Stirring by } \\
\text { particles }\end{array}$ & - & Local & Broad & High & 4 \\
\hline
\end{tabular}

\section{Design and Theoretical Analysis of Micromixer}

The channels in micromixers can be divided into two parts. The first part is a T-shaped or Y-shaped junction, serves as the contacting unit for fluids. The second part is used for further in-line mixing, mixing enhancement or provides the residence time needed for chemical reaction or other transformation. These two parts are shaped according to their tasks. 
Fig. 4.1 shows a schematic diagram of the micromixer geometry. It is a type of Y-junction, square chamber mixer with obstacles in flow path. These obstacles can produce chaotic advection. The variables for the mixing studies are the flow rate corresponding to Re and the channel cross-section area. The mixing processes between the two fluids took place mainly in the narrow channel after they collided with the obstacle producing the turbulence there [1].

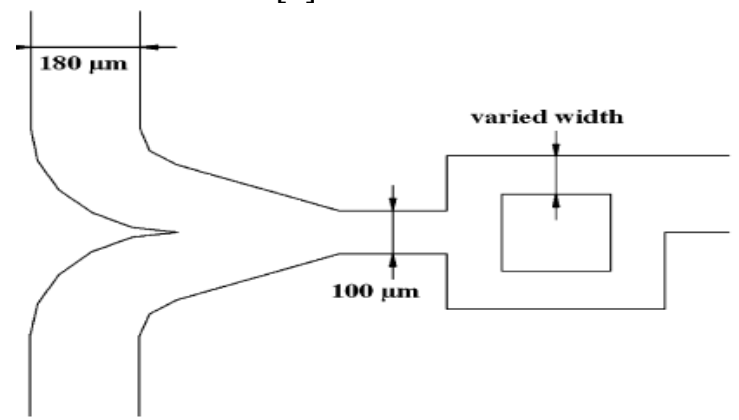

Fig. 4.1 Micromixer with a variable narrow channel width

It is essential to consider two characteristic dimensionless numbers, $\mathrm{Re}$ and the Peclet number $(\mathrm{Pe})$, in order to determine the effective operation condition of a passive micromixer.

Re is defined as,

$\operatorname{Re}=\frac{\rho \vartheta \mathrm{d}}{\mu}$

Where Re represents the ratio between momentum and viscous friction, and $\mu, \rho, v$, and $d$ indicate the dynamic viscosity, fluid density, fluid velocity, and characteristic dimension.

Pe is defined as

$\mathrm{Pe}=\frac{\mathrm{vd}}{\mathrm{D}}$

Where Pe represents the ratio between the mass transport due to convection and diffusion.

The transverse diffusion time can be estimated by the following equation

$\mathrm{t}=\frac{\mathrm{d}^{2}}{\mathrm{D}}$

Where $\mathrm{d}$ is the channel width and D is the diffusion coefficient.

Therefore, the characteristic mixing length (L) of the micromixer to achieve complete mixing is

$L=\vartheta t=\vartheta \frac{d^{2}}{D}=P e d$

In the microfluidic channel, $\mathrm{L}$ is of the order of several centimeters. Eq. (3) therefore indicates that a higher $\mathrm{Pe}$ number is correlated with increased difficulty in achieving mixing by diffusion due to the limited $\mathrm{L}$ value. Therefore, if the Re value is lower than a critical value for turbulent flow or if it is ineffective to induce vortex formation, the higher Re value makes the mixing less efficient. The Re critical value depends on the flow velocity and the mixer structure.

\section{Analysis of Mixing Characteristics}

The mixing based on the chaotic advection principle was only useful if the vortex was formed effectively. The vortex formation commenced at the entrance of the Y-junction when the Re number exceeded the critical Re value. In this study, the vortex formation at the entrance could not be observed due to the low $\mathrm{Re}$ range operation.
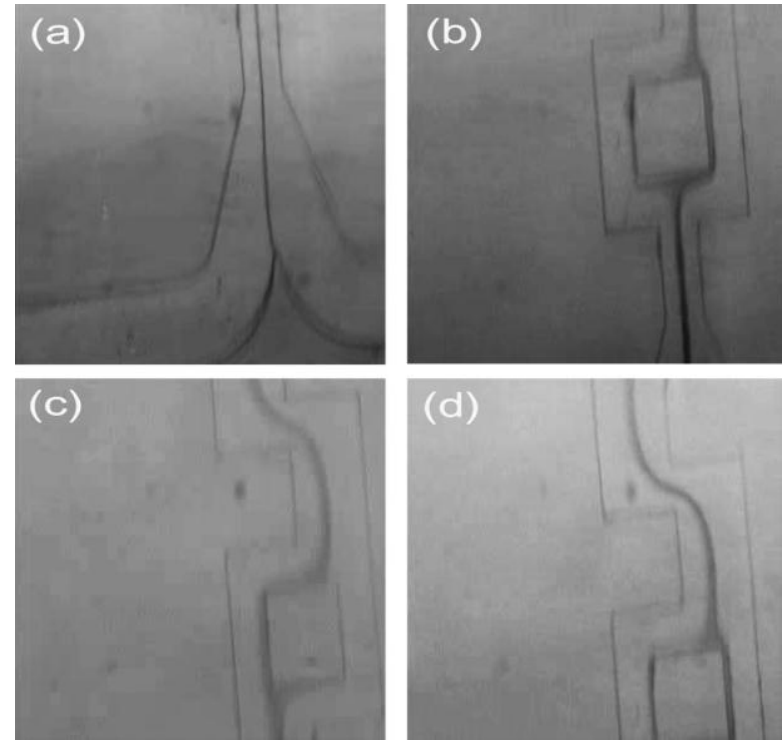

Fig. 5.1 Mixed flow at different position: (a) mixing interface at the entrance at initial time, (b) mixing interface at the entrance with the passage of time, (c) unsymmetrical mixed flow and (d) symmetric mixed flow

In Fig. 5.1(a) no vortex formation was evident at the Yjunction. The two solutions were mixed at the boundary and the mixed flow was in the laminar regime. This mixed flow then collided with the square shape at the center due to the symmetry of the two inlet flows. The thickness of the color flow increased gradually due to the lateral molecular diffusion. Fig. 5.1(b) shows that the line-width of the boundary of mixing at the entrance increased over time due to the lateral diffusion. Therefore, the mixing percentage after the flow passed the stage number 1 also increased with increasing mixing time. Fig. 5.3(c) shows the unsymmetry of the mixed flow due to the bubble formation in the flow direction. This is becoming a serious problem in microscale applications for the mixing process because the bubbles can deform the flow into an unsymmetric shape, thereby reducing the mixing efficiency. Fig. 5.4(d) shows the symmetry of the mixed flow by controlling the two-inlet flows well. It clearly shows that the mixing percentage increased with increasing flow rate due to the amount of vortices 


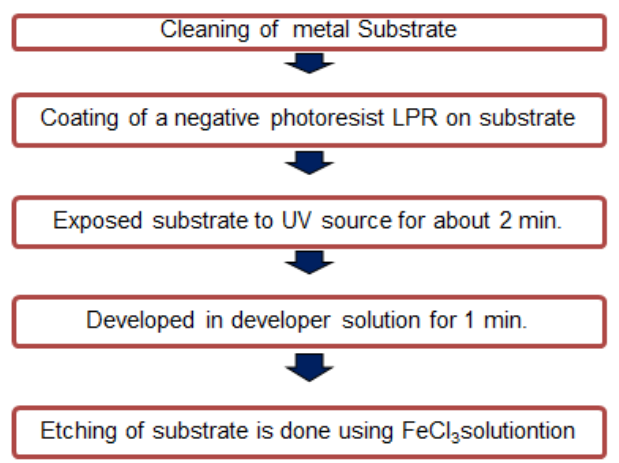

Flow chart A: Photochemical Machining Process

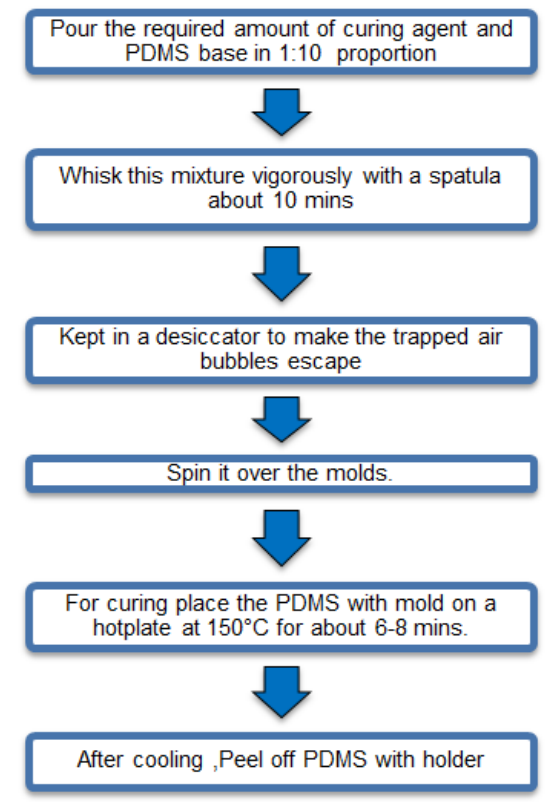

Flow chart A : Photochemical Machining Process

formation while the flow collided with the obstacles. In fact, for a micromixer with the same structure but without obstacle shapes in the flow path, the mixing process would take a greater time and a longer traveling length to obtain the complete mixing .

Different obstacles like rectangular, triangular and circular when placed on the wall of the channel also affect the mixing length of the channel. The shape and size of the obstacles and their location on the wall of the channel also affecting the mixing length.

\section{Fabrication of Microchannels}

The AutoCAD drawing of $\mathrm{Y}$ shape circular and rectangular chamber channel is printed on a transparency sheet which is called mask. Steps required carrying out Photochemical Machining and PDMS Mold making are shown in following flow charts $A$ and $B$ respectively.

\section{Conclusions}

Mixing length in the channel generally depends upon diffusion coefficient, width and height of the channel inlet velocities of the fluids, viscosity of fluids and geometric layout of micro-mixer. As the width of channel increases, the mixing time increases which further increases the mixing length of micro-mixer channel. Increase in diffusion coefficient leads to decrease in mixing length of micro-mixer channel Decreasing the inlet velocities of the incoming fluids, decreasing the mixing length of micro-mixer channel.

\section{References}

T. Nguyen, Min-Chan Kim, Joon-Shik Park and N. E. Lee, An effective passive microfluidic mixer utilizing chaotic advection, Sensors and Actuators B 132 (2008) 172-181.

Alam and Kwang-Yong Kim, Mixing performance of a planar micromixer with circular chambers and crossing constriction channels, Sensors and Actuators B 176 (2013) 639- 652.

L. Michael Ward, Seck Hoe Wong and Christopher W. Wharton, Micro T-mixer as a rapid mixing micromixer, Sensors and Actuators B 100 (2004) 359-379.

Yong Kweon Suh and Sangmo Kang, Mixing in Microfluidics, micromachines

Lorenzo Capretto, Wei Cheng, Martyn Hill, and Xunli Zhang Micromixing within Microfluidic Devices, Top Curr Chem (2011) 304: 27-68. 